


\title{
Estrategias Pedagógicas para Desarrollar las Habilidades Metacognitivas del Alumno de Instrumento con el Fin de Maximizar la Eficacia de sus Prácticas Instrumentales
}

\author{
Malinalli Peral García, Universidad Laval (Canadá) \\ Francis Dubé, Universidad Laval (Canadá)
}

\section{Resumen}

Este artículo trata sobre los alumnado en su desarrollo habilidades metacognitivas de los conocimientos y estrategias metacognitivo. A continuación, estudiantes, las cuales han sido pedagógicas que se pueden se presentan los modos de adaptadas a las necesidades aplicar para desarrollar las intervención pedagógicos a específicas de la práctica habilidades metacognitivas del privilegiar para iniciar al alumno instrumental. Estas herramientas alumno de instrumento. de un instrumento en un proceso ofrecen al músico-pedagogo Primero, se explican los distintos metacognitivo. Finalmente, se referencias para guiar el componentes de la proponen dos estrategias desarrollo de las habilidades metacognición que el profesor de pedagógicas provenientes de las metacognitivas de su alumnado. instrumento debe integrar a su ciencias de la educación pedagogía para guiar a su diseñadas para desarrollar las

\section{Palabras Clave}

Metacognición; práctica instrumental; estrategias de enseñanza; enseñanza instrumental; estudio instrumental.

\section{Pedagogical Strategies to Develop Students' Metacognitive Skills in Order to Optimize their Playing Efficacy}

\section{Malinalli Peral García, Laval University (Canada) Francis Dubé, Laval University (Canada)}

\section{Abstract}

This article is about the in their metacognitive been adapted to the specific knowledge and teaching development are discussed. needs of instrumental practice strategies that can be applied to Then, the pedagogical modes and intended to develop develop instrument students' which should be prioritized to metacognitive skills of students

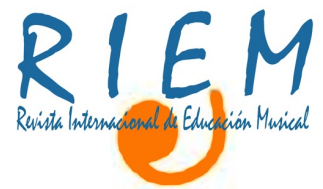

International Society for Music Education ISMEO Established in 1953 metacognitive skills. First, the start instrument students in a will be explained. These tools various components of metacognitive process are offer the musician-teacher metacognition that instrument presented. Finally, two teaching references to guide the teachers must integrate into their strategies coming from the development of metacognitive pedagogy to guide their students educational sciences, which have skills of their students.

\section{Keywords}

Metacognition; instrumental practice; teaching strategies; instrumental strategies; instrumental study. 


\section{$\stackrel{+}{\circ}$ Estrategias Pedagógicas para Desarrollar las Habilidades Metacognitivas del Alumno de Instrumento con el Fin de Maximizar la Eficacia de sus Prácticas Instrumentales}

\section{por Malinalli Peral García y Francis Dubé, Universidad Laval (Canadá)}

Según Flavell (1976), la metacognición hace referencia al conocimiento que una persona tiene sobre sus propios procesos y productos cognitivos. Las personas consideradas como expertas, ya sea en la música o en otros ámbitos profesionales, demuestran poner en práctica más habilidades metacognitivas que los principiantes (Chase \& Simon, 1973; Ericsson, Krampe \& Tesch-Romer 1993; Hallam, 2001). En el ámbito musical, este tipo de habilidades desempeña un papel fundamental durante la adquisición y el desarrollo de competencias musicales, especialmente porque le permiten al músico ser más eficaz durante sus periodos de estudio instrumental (Hallam, 2001; Barry \& Hallam, 2002).

Efectivamente, los músicos profesionales organizan sus practicas en función de los errores detectados con el fin de corregirlos (Hallam, 1997, Op. Cit. en Woody, 2001), mientras que los estudiantes de música, especialmente los principiantes, pueden pasar hasta 90\% del tiempo practicando la pieza de principio a fin sin utilizar estrategias concretas que mejoren su ejecución (McPherson \& Renwick, 2001). Ahora bien, el músico puede vigilar y corregir sus propios errores (Kenny, 1992, Op. Cit. en Woody, 2001) de manera eficiente gracias, justamente, a la adquisición de habilidades metacognitivas. Dada su importancia durante el aprendizaje instrumental, sería primordial desarrollar dichas habilidades en el alumno de instrumento para mejorar la calidad de su práctica.

A nivel pedagógico, el profesor de instrumento puede desempeñar un papel crucial en el desarrollo de las habilidades metacognitivas de sus estudiantes. Para ello, éste debe incitarlos a reflexionar sobre la forma de estudiar, aprender y memorizar su repertorio, insistiendo particularmente en cómo podrían integrar este enfoque reflexivo a sus conocimientos musicales (Aiello \& Williamon, 2002). Sin embargo, para trabajar en este sentido, el profesor debe poseer cualidades específicas y poner en práctica estrategias pedagógicas relacionadas concretamente con el desarrollo de las habilidades metacognitivas del alumno (Lafortune, Jacob \& Hébert, 2000; Doly, 1997), tema sobre el que fundamentalmente se hablará en este artículo.

Así pues, en primer lugar se definirá qué es la metacognición y cuáles son los componentes que la integran. Luego se describirán las habilidades metacognitivas observadas con más frecuencia en el músico experto, para luego relacionarlas con las que se deben desarrollar en el estudiante de instrumento. Por último se presentarán métodos de intervención y estrategias pedagógicas para contribuir al desarrollo de las habilidades metacognitivas del alumno instrumentista.

\section{Definición y componentes de la metacognición}

Desde principios del siglo XX, diversos autores han llevado a cabo investigaciones científicas y escritos teóricos sobre los procesos relacionados con la metacognición ${ }^{1}$. Sin embargo, Flavell es a menudo considerado el pionero de estas investigaciones (Saint-Pierre, 1994), gracias a la que efectuó sobre la meta-memoria ${ }^{2}$ y que lo llevó a proponer la primera definición de este concepto. Según él, la metacognición:

Se refiere al conocimiento que una persona tiene sobre sus propios procesos y productos cognitivos o cualquier otra cosa relacionada con ellos [...]. Por ejemplo, yo hago metacognición $[\ldots]$ si me doy cuenta de que tengo más problemas para aprender $\mathrm{A}$ que $\mathrm{B}$; si creo que debo comprobar $\mathrm{C}$ antes de aceptarlo como un hecho [...]. La metacognición se refiere, entre otras cosas, a la observación activa seguida de la regulación y la orquestación de estos procesos [...] generalmente con el fin de lograr un propósito o un objetivo concreto (Flavell, 1976, p. 232).

Así, la metacognición consta de dos componentes principales, a saber: los conocimientos metacognitivos (Flavell, 1979; 1987) y el uso y adaptación de estos conocimientos para coordinar los procesos mentales (Brown, 1987).

\section{Los conocimientos metacognitivos}

Los conocimientos metacognitivos están agrupados en tres categorías: los conocimientos acerca de las personas, de las tareas y de las estrategias.

\section{Conocimientos acerca de las personas}

Los conocimientos acerca de las personas se pueden dividir en tres sub-categorías: intra-individuales, interindividuales y universales (Flavell, 1987). Los intraindividuales tienen que ver con los conocimientos o las creencias que el individuo tiene sobre su propio funcionamiento cognitivo. Por ejemplo, saber que se tienen dificultades para leer la música contemporánea o que el estudio instrumental es más eficaz por las mañanas que por las tardes.

Los conocimientos interindividuales se refieren a aquellos en los que el individuo se compara con los demás. Por ejemplo, cuando un músico es consciente de que puede tocar mejor el repertorio más virtuoso que otros músicos de su generación.

Los conocimientos universales hacen alusión a las representaciones que cada individuo tiene sobre los aspectos generales de la cognición humana. Se puede tratar, entre otros, de conocimientos o intuiciones sobre el funcionamiento de la mente, de la memoria o de la inteligencia. Por ejemplo, saber que la memoria a corto plazo puede tratar alrededor de siete elementos a la vez $( \pm 2)$ a partir de los 14 años.

\section{Conocimientos acerca de las tareas}

Los conocimientos acerca de las tareas se refieren a la naturaleza misma de la tarea así como a los requisitos necesarios para llevarla a cabo. Se trata de conocimientos adquiridos sobre las diferentes informaciones relacionadas con experiencias pasadas y sobre el proceso que cada una de ellas necesita para su ejecución (Flavell, 1987). Un ejemplo de este tipo de conocimientos sería que el músico debe ser 
consciente de que hay cuatro tipos de memoria que intervienen en el proceso de memorización de una pieza -auditiva, visual, kinestésica, conceptual-, pero que es preferible concentrar el aprendizaje de memoria utilizando la memoria conceptual, porque ésta ayuda al instrumentista a sentirse más seguro cuando toca sin la partitura (Dubé, 2006).

\section{Conocimientos acerca de las estrategias}

Los conocimientos acerca de las estrategias se refieren a los diferentes procedimientos que se utilizan para alcanzar un objetivo específico. Dependiendo del objetivo, estas estrategias se pueden clasificar en estrategias cognitivas y estrategias metacognitivas. Así, las estrategias cognitivas le permiten al individuo lograr un objetivo cognitivo, por ejemplo, leer una partitura con el fin de descifrarla. En cuanto a las estrategias metacognitivas, ellas sirven para comprobar si el resultado es satisfactorio o no, por ejemplo, leer la misma partitura pero esta vez para verificar que no se aprendió ninguna nota errónea.

Según Flavell (1987), los conocimientos acerca de las personas, las tareas y las estrategias siempre están interactuando entre sí y esta interacción se aprende de la misma manera que los conocimientos metacognitivos. De hecho, los conocimientos metacognitivos no difieren de otros tipos de conocimientos almacenados en la memoria a largo plazo, ya que éstos se pueden activar de forma voluntaria (Flavell, 1979). Sin embargo, también es posible que se activen de forma automática e involuntaria durante la actividad cognitiva, y esto, de manera más o menos consciente.

Según Brown (1987), los conocimientos metacognitivos son relativamente estables y se pueden expresar verbalmente, es decir, la persona puede expresar en voz alta lo que piensa sobre su proceso de aprendizaje, de las estrategias más eficientes, etc. Sin embargo, este conocimiento puede ser cierto o erróneo. Por ejemplo, si una persona cree que debe relacionar un nuevo aprendizaje a los conocimientos que ya están almacenados en la memoria a largo plazo (conocimiento metacognitivo sobre las personas/universal) para asimilarlo efectivamente, se trata de un conocimiento cierto. Al contrario, si una persona cree que para llegar a tocar una pieza rápidamente se debe estudiar siempre aumentando gradualmente la velocidad del gesto (conocimiento metacognitivo sobre las estrategias) estamos hablando de un conocimiento erróneo (Jørgensen, 2004). Cabe añadir que los conocimientos metacognitivos se incrementan con la edad. Este tipo de conocimientos requiere que el estudiante tenga una visión retrospectiva y considere sus propios procesos cognitivos como objetos de pensamiento y reflexión (Brown, 1987).

\section{Las experiencias metacognitivas}

Flavell (1979) afirma que el conocimiento metacognitivo puede conducir a experiencias cognitivas o afectivas conscientes llamadas experiencias metacognitivas. Estas experiencias se diferencian de otros tipos de experiencias en que tienen una relación específica con la actividad intelectual (Flavell, 1987). Por ejemplo, se trata de una experiencia metacognitiva cuando una persona es consciente de que se siente ansiosa si no comprende algo que quiere saber. Con la edad, la persona aprende a interpretar este tipo de experiencias y a reaccionar ante ellas adecuadamente, a diferencia de los niños pequeños que a menudo, las viven conscientemente pero sin ser capaces de interpretarlas correctamente (Flavell, 1987).

Asimismo, Flavell (1987) explica que el individuo es probablemente más propenso a vivir experiencias metacognitivas cuando:

- La situación lo pide explícitamente.

- La situación no es completamente nueva ni completamente familiar, es decir, cuando la persona conoce la situación lo suficiente como para estar confundido y ser capaz de formular preguntas pero no tanto como para que el proceso sea totalmente automático y sin esfuerzo.

- La persona hace inferencias, juzga y toma las buenas decisiones.

- La actividad cognitiva se halla en el centro de la resolución de un problema.

- La atención y los recursos nemotécnicos de la persona no se ven disminuidos por experiencias subjetivas más urgentes como el miedo, la ansiedad o la depresión.

Las experiencias metacognitivas juegan igualmente un papel importante en el proceso de aprendizaje, ya que, de acuerdo con Flavell (1979), éstas pueden tener un efecto decisivo sobre las tareas o los objetivos cognitivos perseguidos, así como sobre las estrategias y los conocimientos empleados. En otras palabras, cuando el individuo vive la experiencia metacognitiva, éste puede fijarse nuevos objetivos, así como revisar o abandonar los establecidos en un principio. Este tipo de experiencia puede incluso afectar la base de los conocimientos metacognitivos. Por ejemplo, el músico que se siente ansioso cuando se da cuenta de que su manera de estudiar no le conduce a los resultados deseados (experiencia metacognitiva) puede decidir cambiar de estrategia con el fin de lograrlo. De hecho, la interacción de la movilización de los conocimientos metacognitivos con las experiencias metacognitivas daría lugar a la coordinación de los procesos mentales.

\section{La coordinación de los procesos mentales}

El segundo componente principal de la metacognición es la coordinación de los procesos mentales. Esto es a lo que Lafortune, Jacob \& Hébert (2000) llaman habilidades metacognitivas. Para estos autores:

Una habilidad metacognitiva es la capacidad de movilizar sus conocimientos y experiencias, pero con la intención explícita de planear la ejecución de una tarea para supervisarla mejor, evaluarla y emitir juicios críticos sobre la eficacia de la manera de proceder tomando en cuenta las estrategias utilizadas y el objetivo establecido. Este juicio no sólo permite enriquecer sus conocimientos metacognitivos, sino también desarrollar un conocimiento consciente que podrá ser utilizado en situaciones cada vez más complejas. Las principales manifestaciones de una habilidad metacognitiva son el control y la regulación del proceso de aprendizaje, ya que son el resultado de una evaluación constante y consciente y que por lo tanto favorecen la reutilización. (Lafortune, Jacob \& Hébert, 2000, p. 12-13).

Así, las habilidades metacognitivas le permiten al individuo usar y adaptar sus propios conocimientos metacognitivos con el fin de coordinar su actividad mental. Éstas constan de tres tipos de actividades relacionadas con la ejecución de una tarea: las actividades de planificación, de control y de regulación.

\section{Las actividades de planificación}

Las actividades de planificación permiten establecer objetivos y escoger estrategias para alcanzarlos, así como 
determinar los parámetros de evaluación que se utilizarán durante y al final de la ejecución de la tarea. Por ejemplo, un músico realiza actividades de planificación cuando, al comenzar su práctica instrumental decide cómo solucionar un pasaje técnico seleccionando estrategias específicas, determinando cuánto tiempo deberá estudiar ese pasaje para lograr su objetivo, y cuándo y de qué forma va a autoevaluarse durante y al final del estudio para lograr su objetivo.

\section{Las actividades de control}

Las actividades de control tienen como objetivo vigilar y verificar la eficacia de la actividad cognitiva en curso. Éstas consisten principalmente en la evaluación de las acciones realizadas durante el aprendizaje. Así, preguntarse si la estrategia seleccionada para dominar una dificultad instrumental está dando los resultados deseados es un ejemplo de actividad de control.

\section{Las actividades de regulación}

Las actividades de regulación permiten modificar el proceso utilizado durante la actividad cognitiva una vez realizadas las actividades de control. Por ejemplo, se puede decidir al inicio de la práctica conservar la estrategia seleccionada para solucionar un problema técnico, pero adaptarla disminuyendo la velocidad del gesto prevista inicialmente, para solucionarlo más eficazmente.

Según Brown (1987), las actividades de planificación, de control y de regulación son relativamente inestables ya que varían en función de la tarea y de la situación de aprendizaje en la que ésta es realizada. Además, no siempre se pueden expresar verbalmente, porque saber cómo hacer algo no significa necesariamente que la persona realice la actividad conscientemente ni que pueda explicar su acción con palabras. Estas actividades también son relativamente independientes de la edad ya que todo aprendizaje activo requiere una forma de auto-regulación. De hecho, los niños pequeños regulan y supervisan tanto como los adultos sus propias actividades de aprendizaje.

La figura 1 presenta, a modo de resumen, una síntesis de las nociones metacognitivas explicadas hasta ahora:

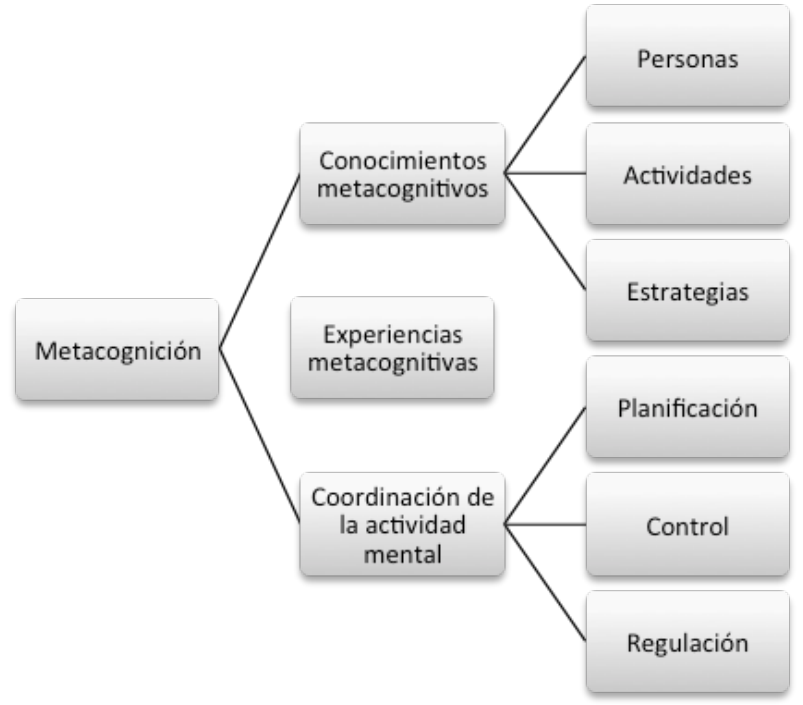

Figura 1: Nociones metacognitivas.

\section{El músico experto y el profesor guía de la metacognición}

Habilidades metacognitivas del músico experto

Las investigaciones sobre la adquisición de la experiencia sugieren que las personas con una gran experiencia en un área específica poseen muchas más habilidades metacognitivas que los principiantes (Chase \& Simon, 1973; Ericsson, Krampe \& Tesch-Romer, 1993; Hallam, 2001). Así pues, los expertos demuestran una capacidad analítica que les permite identificar más fácil y rápidamente los problemas que tienen que resolver (Hallam, 2001). Además, adaptan su manera de actuar a las restricciones inherentes de la tarea (Holyoak, 1991), utilizan más variedad de estrategias para mejorar su objetivo y modifican estas estrategias en función de los errores identificados (Chase \& Ericsson, 1981; VanLehn, 1991, Op. Cit. en Ericsson, Krampe \& TeschRömer, 1993). Por último, los expertos presentan capacidades superiores en la manera de utilizar su memoria ${ }^{3}$ y su autorregulación (Glaser \& Chi, 1988). De otro modo, el experto es capaz de hacer lo correcto en el momento adecuado (Dorner \& Schölkopf, 1991, Op. Cit. en Holyoak 1991).

En el ámbito musical, la experiencia se adquiere practicando el instrumento a diario, y las habilidades metacognitivas llevadas a cabo por el músico modulan la eficacia de los periodos de estudio. Esto es justamente lo que lleva a Hallam (2001) a afirmar que el músico debe tener habilidades metacognitivas para realizar periodos de estudio eficaces:

Para ser capaz de reconocer la naturaleza y las necesidades de una tarea específica; de identificar problemas concretos; de poseer una serie de estrategias para hacer frente a estas dificultades; de saber qué estrategias son apropiadas para realizar cada tarea; de vigilar el progreso hacia la obtención del objetivo. Si el progreso no es satisfactorio, se debe reconocer y utilizar estrategias alternativas; evaluar los resultados del aprendizaje en situaciones en las cuales se llevará a cabo la prestación musical y tomar las medidas necesarias para mejorar el resultado en el futuro. (Hallam, 2001, p. 28)

Además, según Hallam (2001), las habilidades metacognitivas del músico también deben incluir herramientas para administrar el tiempo de la práctica para poder, entre otras cosas, cumplir con ciertos plazos profesionales o mantener el nivel de concentración y de motivación. Dicho de otro modo, las habilidades metacognitivas son necesarias, incluso esenciales, para la optimización del estudio individual del músico. Así pues, las investigaciones llevadas a cabo por Hallam (2001) indican que los músicos expertos tienen más habilidades metacognitivas que los principiantes, y que estas habilidades se observan en cómo llevan a cabo la práctica instrumental. Por consiguiente, Hallam (2001) explica que los músicos expertos que han desarrollado habilidades metacognitivas:

- Son conscientes de sus propias fuerzas y debilidades (conocimiento acerca de las personas) ${ }^{4}$, lo que les permite identificar, durante la lectura de nuevo repertorio, los pasajes que requieren más trabajo en las sesiones posteriores (planificación).

- Tienen un amplio conocimiento acerca de la naturaleza de las diferentes tareas -leer un nuevo repertorio, organizar el estudio, trabajar los pasajes 
difíciles, mantener la concentración, preparar una presentación-, informaciones que son necesarias para poder realizarlas con éxito (conocimientos acerca de las tareas).

- Conocen una variedad de estrategias que pueden ser utilizadas de acuerdo a sus necesidades específicas y a la tarea (conocimientos acerca de las estrategias).

- Orientan su trabajo en función de objetivos concretos (planificación).

- Tienen una base de conocimientos que les permite evaluar las exigencias de la tarea, identificar los problemas (conocimientos acerca de las tareas), reconocer sus errores, evaluar el progreso (actividades de control) y tomar las medidas necesarias para solucionar los problemas (actividades de regulación).

Dicho esto, se puede suponer que el profesor de música, siendo él mismo un músico experto, es capaz de ayudar al alumno a desarrollar dichas habilidades metacognitivas para que pueda efectuar prácticas cada vez más eficaces con el instrumento. Ahora bien, para hacerlo, es importante que el profesor tenga cualidades pedagógicas específicas para desarrollar las habilidades metacognitivas de su alumnado y que pueda incluir en su enseñanza estrategias para favorecer su adquisición.

\section{Cualidades del profesor guía de la metacognición}

Las investigaciones sobre el desarrollo de la metacognición realizadas por Lafortune, Jacob \& Hébert (2000) muestran que, para favorecer el desarrollo de habilidades metacognitivas de los alumnos, el profesor debe estructurar su enseñanza en consecuencia y poseer cualidades pedagógicas específicas. Primero debe conocer los fundamentos psicológicos relacionados con la metacognición ${ }^{5}$ y construir estrategias de enseñanza diseñadas explícitamente para desarrollarlas. También debe proponer constantemente momentos de reflexión para así favorecer el surgimiento de comentarios metacognitivos por parte de los estudiantes y suscitar la verbalización de sus procesos mentales. Por último, debe ser capaz de reconocer los «desbloqueos metacognitivos» del estudiante con el fin de despertar en él una toma de conciencia de sus procesos mentales.

Por su parte, Doly (1997) sostiene que el experto debe evitar sobrecargar cognitivamente al alumno durante su aprendizaje. Para ello, la tarea en cuestión:

Debe ser lo suficientemente simple como para permitirle al individuo partir de lo que ya sabe, de sus propios conocimientos y habilidades actuales y embrionarias, pero al mismo tiempo lo suficientemente difícil como para presentar un problema que le obligue a tomar riesgos para ir más lejos de lo que puede hacer solo" (ibíd, p. 59).

El docente experto también debe saber cómo utilizar positivamente los errores cometidos por el alumno. Para hacerlo, puede ayudarle a identificarlos y analizarlos para llevarlo a "tomar consciencia de los procedimientos y conocimientos que utilizó de manera espontánea y que lo condujeron al error, para permitirle después la construcción de estrategias de solución, y, eventualmente y de manera más general, estrategias para vencer el fracaso" (ibíd., p. 61-62).

De la misma manera, según este mismo autor (ibíd.), el profesor debe proponer actividades que sean significativas para el alumno, es decir, situaciones de aprendizaje en las que éste pueda hacerse una idea del objetivo que se quiere lograr a partir de criterios de evaluación que le permitan guiar sus actividades. Dicho de otro modo, el profesor debe fomentar en los alumnos una motivación intrínseca que les ayude a realizar la tarea, motivación que será la clave para llevarla a cabo con éxito.

Por último, el profesor debe evitar proporcionar ayuda adicional al alumno que ya puede autorregularse o lograr su aprendizaje con satisfacción, no sólo porque este tipo de ayuda es inútil para él, sino porque podría incluso inhibir habilidades o estrategias ya desarrolladas o en proceso de serlo (ibíd.).

\section{Estrategias pedagógicas para desarrollar las habilidades metacognitivas de los alumnos.}

En esta sección se proponen dos estrategias pedagógicas que pueden favorecer el desarrollo de las habilidades metacognitivas del alumno instrumentista. Se trata de la estrategia en tres fases y del cuaderno diario de las prácticas.

\section{Estrategia en tres fases}

Lafortune, Jacob \& Hébert (2000) explican que "el desarrollo cognitivo y metacognitivo es un proceso gradual de interiorización y personalización a través de la interacción social" (p. 16). Este proceso de interiorización es un acto de reflexión complejo en el cual el alumno mantiene conscientemente un diálogo interno sobre la tarea que realiza. Sin embargo, este proceso no es automático y requiere la mediación de un guía con experiencia para llevarlo a cabo adecuadamente.

La mediación hace referencia a la manera en la que un experto organiza el entorno físico y mental de otro individuo con menos experiencia para que pueda pensar mejor, actuar y construir su identidad (Doly, 1997). Esta mediación entre el experto y el alumno parece incluso ser una condición sine qua non para que el estudiante inicie el proceso de interiorización requerido para desarrollar sus habilidades metacognitivas. Según Doly (1997), este proceso de interiorización está basado en cuatro etapas distintas:

1. Para empezar, el experto debe tener los conocimientos y las habilidades necesarias para coordinar la tarea.

2. A continuación, el experto debe poner en práctica los conocimientos y habilidades en interacción con el principiante mediante el lenguaje, a través de palabras de aliento, de indicaciones, de invitaciones a evaluarse.

3. Después, el principiante debe aprender a usar cada vez más, los conocimientos y habilidades intencionadamente en compañía del experto.

4. Por último, el principiante debe llegar a gestionar de forma autónoma su actividad sin el experto.

En un contexto educativo, estos pasos corresponden, según Lafortune, Jacob y Hébert (2000), al modelado (punto 1), la práctica guiada (puntos 2 y 3 ) y la práctica independiente (punto 4). La aplicación de la secuencia propuesta por estos autores fue llevada a cabo en la enseñanza instrumental para construir nuestra estrategia pedagógica $^{6}$.

\section{El modelado}

El modelado corresponde a la primera etapa del proceso de interiorización. Aquí el profesor ofrece al alumno un modelo de realización de una tarea a partir de sus 
conocimientos personales. Durante esta fase, el profesor explica en voz alta su proceso mental al mismo tiempo que ejecuta la tarea. El objetivo del profesor es hacer visible el diálogo interno, mostrando los procedimientos mentales que se deben poner en marcha para conseguir realizar la tarea correctamente.

Durante esta fase, el profesor debe estimular primero los conocimientos previos del alumno que puedan ser pertinentes para realizar la tarea. Este enfoque le permitirá comparar su manera de realizarla con la del profesor. A continuación, el profesor expresa en voz alta cómo realiza la tarea, así como cada una de las ideas que le vienen a la cabeza cuando la está ejecutando. Por ejemplo, verbaliza las preguntas que se hace, las acciones que realiza para vigilar la actividad, evaluarla, adaptar su enfoque, etc.. En otros términos, el profesor no se conforma únicamente con dar un ejemplo, sino que se presenta a sí mismo como tal.

Para ilustrar este concepto educativo a partir de un ejemplo, se puede tomar el aprendizaje postural instrumental. El profesor podría modelar la adquisición de una buena postura con el instrumento, verbalizando delante del alumno todos los elementos que deben ser tomados en cuenta para lograrla al mismo tiempo que realiza la acción. Asimismo, expresa en voz alta los ajustes necesarios que realiza mientras trata de lograr la posición instrumental deseada, explicándole también las razones que justifican estos ajustes.

\section{La práctica guiada}

La práctica guiada corresponde a la segunda y tercera etapas del proceso de interiorización de Doly (1997) que el alumno debe seguir para poner en marcha un proceso de aprendizaje metacognitivo. Aquí, el profesor invita al alumno a verbalizar su manera de proceder siguiendo el modelo realizado antes por el profesor. A continuación, con preguntas e incentivos, el profesor ayuda al alumno a planificar su actividad, dirige su atención hacia los aspectos importantes para una buena ejecución, y lo lleva a autoevaluarse y corregirse.

Si tomamos el ejemplo postural anterior, durante esta fase el profesor invitaría al alumno a verbalizar sus pensamientos mientras intenta aplicar a su interpretación la actitud postural modelada anteriormente por el profesor. Cuando éste se dé cuenta de que el alumno ha omitido ciertos elementos, le motiva y le hace preguntas para dirigir su atención hacia los elementos omitidos. El profesor también puede volver a la fase de modelado para hacer más hincapié en los elementos que requieran más atención del alumno, antes de continuar con la práctica guiada.

\section{La práctica independiente}

La práctica independiente corresponde a la última etapa del proceso de interiorización, es decir, es la etapa durante la cual el alumno practica sin la presencia del experto. En principio, durante esta etapa el alumno debe ser capaz de mantener una reflexión interna mientras coordina la tarea, así como de hacerse las preguntas adecuadas para realizarla correctamente. De hecho, el alumno reproduce la interacción que tuvo lugar entre él y el experto durante la práctica guiada, pero esta vez realizando a la vez el papel del experto y el del alumno.

\section{El diario de prácticas}

La segunda estrategia que proponemos para desarrollar las habilidades metacognitivas del alumno consiste en la realización de un diario de prácticas en el que el alumno escribe sus observaciones sobre sus conocimientos y sus habilidades metacognitivas. Utilizando la escritura como medio reflexivo, el alumno observa su pensamiento interno y deja las huellas que le permiten, más tarde, evaluar su progreso y su manera de aprender (Lafortune, Jacob \& Hébert, 2000). Ahora bien, el hecho de escribir no desarrolla automáticamente las habilidades metacognitivas. Para que sea efectivo, el profesor debe guiar las observaciones del alumno de forma estructurada al tiempo que busca alcanzar objetivos específicos. Dicho de otro modo, el maestro debe "proponer actividades de escritura que permitan al alumno tomar consciencia de la gestión y regulación de su proceso de aprendizaje" (Lafortune, Jacob \& Hébert, 2000, p. 71).

Para tener un referente de cómo orientar el proceso de reflexión de los alumnos, se adaptó el cuestionario sobre los conocimientos metacognitivos y la coordinación de la actividad mental (COMÉGAM), diseñado y validado por Richer y otros (2004), a las necesidades de la práctica instrumental. Construido para medir y desarrollar la metacognición, este cuestionario (véase el Anexo A) también puede guiar las acciones pedagógicas del profesor instrumental que desea llevar al estudiante a desarrollar sus habilidades metacognitivas. Esta aplicación contiene afirmaciones relacionadas con los conocimientos metacognitivos (personas, tareas, estrategias) y la coordinación de la actividad mental (planificación, control, regulación) en un contexto de práctica instrumental.

El cuestionario consta de 36 enunciados y se divide en seis secciones. Cada sección corresponde a un aspecto específico de la metacognición. Dado que las afirmaciones de la versión original fueron diseñadas para el aprendizaje en un medio escolar, la modificación de estas afirmaciones fue necesaria con el propósito de adaptarlas al contexto de aprendizaje instrumental. Por ejemplo, en la versión original, el primer enunciado dice: conozco cuáles son las estrategias que más me ayudan a aprender. Este enunciado fue reemplazado por: Conozco cuáles son las estrategias que más me ayudan a practicar.

Una vez que el profesor de instrumento identificó, con la ayuda del cuestionario, los componentes sobre los que el alumno debe concentrarse para desarrollar sus habilidades metacognitivas, se pueden entonces utilizar las fichas de reflexión (véase el Anexo B) para guiar las observaciones que el alumno anotará en su diario. Aquí también se modificaron las afirmaciones de la versión original de dichas fichas con el fin de adaptarlas al contexto de práctica instrumental. Cada ficha ha sido diseñada para hacer que el alumno reflexione sobre un aspecto metacognitivo específico. Éstas se componen principalmente de preguntas y de pistas de reflexión para guiar al alumno instrumentista en su proceso metacognitivo.

Se propone que el profesor de instrumento utilice este cuestionario y las fichas de reflexión de la manera siguiente:

- Al inicio del ciclo escolar, el alumno responde el cuestionario con el fin de identificar cuáles son los aspectos metacognitivos que necesitan un mayor desarrollo.

- A lo largo del año, el profesor guía al alumno en sus observaciones usando las fichas de reflexión. El alumno describe después sus observaciones en su diario. 
- El profesor invita al alumno a compartir sus reflexiones con el fin de proporcionarle información relevante al respecto y con el objetivo de ayudarle a desarrollar sus habilidades metacognitivas y mejorar sus periodos de estudio.

El procedimiento explicado anteriormente busca principalmente hacer que los alumnos reflexionen sobre sus periodos de estudio instrumental para que sean cada vez más eficaces. Sin embargo, las fichas de reflexión también pueden ser utilizadas durante las clases en función de una obra que el alumno esté trabajando con su profesor. Por ejemplo, si un alumno guitarrista debe estudiar un pasaje con un motivo de arpegios que debe tocarse rápidamente, el profesor puede consultar la ficha 1 (Anexo B) sobre las estrategias y adaptarla de esta manera:

1. El enunciado original: ¿hay acciones que deban ser realizadas antes de empezar a estudiar? se puede adaptar así: $\therefore$ Hay acciones que deban ser realizadas antes de empezar a estudiar este motivo de arpegio?

2. La frase original $c^{\text {hay }}$ procedimientos que podrian ayudarte durante tu estudio? se puede cambiar en este caso por ¿hay procedimientos que podrían ayudarte durante tu estudio para poder tocar este motivo de arpegio más rápido?"

3. Por último, la declaración original: para ti, ¿hay momentos más propicios para estudiar? puede convertirse en: para ti, ¿hay momentos más propicios para practicar la velocidad requerida en la mano derecha para tocar este motivo de arpegio correctamente?

\section{Conclusión}

De acuerdo con la literatura especializada, el músico experto tendría más habilidades metacognitivas que el alumno instrumentista. Efectivamente, el experto demuestra una capacidad de análisis que le permite identificar más fácil y rápidamente los problemas que deben ser solucionados, mientras que el músico menos experimentado, entre ellos el músico que comienza su aprendizaje, tiene tendencia a practicar de manera repetitiva sin una visión reflexiva sobre sus errores ni sobre las estrategias necesarias para corregirlos. Por el contrario, si el profesor de instrumento proporciona un acompañamiento pedagógico adecuado, éste puede mejorar las habilidades metacognitivas del alumno, lo que tendría un efecto positivo en la calidad de sus periodos de estudio individual.

Ésta es precisamente la razón que motivó la redacción de este artículo, ya que se quería exponer al profesor de instrumento los conocimientos necesarios para trabajar con mayor eficacia en este sentido así como proponerle una adaptación de los materiales validados científicamente para complementar su actividad pedagógica a nivel metacognitivo. Para ello, primero se explicaron cuáles son los diferentes componentes relacionados con la metacognición y se propusieron dos estrategias pedagógicas para orientar al profesor de instrumento en el desarrollo de las habilidades metacognitivas de los estudiantes. También se adaptó un cuestionario y unas fichas de reflexión ya existentes en la literatura científica. El cuestionario tiene como objetivo identificar las fuerzas y debilidades metacognitivas de los alumnos, mientras que las fichas de reflexión tienen como objetivo orientar eficientemente a los alumnos en su proceso metacognitivo.
El uso de estrategias para promover el desarrollo de la metacognición dentro del marco de la enseñanza instrumental individual es esencial para maximizar la eficacia de las prácticas del alumno. Según nuestro punto de vista, las herramientas y los enfoques pedagógicos propuestos en este artículo pueden ayudar al profesor de instrumento a desarrollar las habilidades metacognitivas de los alumnos de manera más eficiente. El cuestionario y las fichas de reflexión adaptados a la enseñanza instrumental podrían incluso retomarse para llevar a cabo investigaciones en esta área con el fin de evaluar la eficacia real del desarrollo metacognitivo del alumno instrumentista. Es esencial continuar las investigaciones en este ámbito, debido a que la adquisición de habilidades metacognitivas es un componente de gran importancia para mejorar la calidad de los periodos de estudio del alumno instrumentista.

\section{Notas}

${ }^{1}$ Para obtener más información, véase Brown (1987). El autor presenta un breve historial sobre el concepto de metacognición.

${ }^{2}$ Flavell fue el primero en utilizar el término meta-memoria. Véase Flavell (1971).

${ }^{3}$ Holyoak (1991) argumenta que este postulado es especialmente cierto cuando el área de experiencia exige realmente el rendimiento de la memoria.

${ }^{4}$ La identificación de las habilidades descritas entre paréntesis por los autores del artículo especifica los componentes de la metacognición tratados en el texto de Hallam (2001).

${ }^{5}$ Esto es lo que nos motivó a explicar y agrupar en la primera sección del artículo diferentes conocimientos relacionados con la metacognición provenientes de fuentes científicas diversas.

${ }^{6}$ El uso de esta estrategia debe ser utilizada de preferencia con niños de edad escolar, ya sea de manera individual o en grupo. Sin embargo, para la enseñanza en grupo, el profesor puede añadir la fase de la práctica cooperativa - como lo propone Lafortune, Jacob y Hébert (2000, p. 18)- entre la práctica guiada y práctica independiente. Durante esta fase, el profesor invita a los alumnos a colocarse por parejas, con el fin de estimular el intercambio entre ellos sobre la manera de llevar a cabo sus actividades de aprendizaje.

\section{Referencias citadas}

Aiello, R. \& Williamon, A. (2002). Memory. En R. Parncutt \& G. E. McPherson (Ed.). The science and psychology of music performance. Oxford: Oxford University Press.

Barry, N. H. \& Hallam, S. (2002). Practice. En R. Parncutt \& G. E. McPherson (Ed.). The science and psychology of music performance. Oxford: Oxford University Press.

Brown, A. (1987). Metacognition, executive control, selfregulation and other more mysterious mechanisms. En F. E. Weinert \& R. H. Kluwe (Ed.). Metacognition, motivation and understanding. Hillsdale: Lawrence Erlbam.

Chase, W. G. \& Simon, H. A. (1973). The mind's eye in chess. En W. G. Chase (Ed.). Visual information processing. Nueva York: Academic Press.

Doly, A. M. (1997). Métacognition et médiation. Auvernia: GDPR.

Dorner, D. \& Scholkopf, J. (1991). Controlling complex systems. En K. A. Ericsson \& J. Smith (Ed.). Toward a 
general theory of expertise: Prospects and limits. Cambridge: Cambridge University Press.

Dubé, F. (2006). Les repères microstructuraux dans l'apprentissage mnémonique de partitions de piano (tesis doctoral). Montreal: Universidad Laval.

Ericsson, K. A., Krampe, R. T. \& Tesch-Romer, G. (1993). The role of deliberate practice in the acquisition of expert performance. Psychological Review, 100, 363-406.

Holyoake, K. J. (1991). Symbolic connectionism: Towards third-generation theories of expertise. En K. A. Ericsson \& J. Smith (Ed.). Toward a general theory of expertise: Prospects and limits. Cambridge : Cambridge University Press.

Flavell, J. H. (1987). Speculations about the nature and developement of metacogniiton. En F. E. Weinert \& R. H. Kluwe (Ed.). Metacognition, motivation and understanding. Hillsdale: Lawrence Erlbam.

Flavell, J. H. (1971). First discussants comments: What is memory development the development of? Human Development, 14, 272-278.

Flavell, J. H. (1976). Metacognitive aspects of problemsolving. En L. B. Resnick (Ed.). The nature of intelligence. Hillsdale: Lawrence Erlbaum.

Flavell, J. H. (1979). Metacognition and cognitive monitoring. A new area of cognitive-developmental inquiry. American Psychologist, 34(10), 906-911.

Glaser, R. \& Chi, M. T. H. (1988). Overview. En M. T. H. Chi, R. Glaser \& M. J. Farr (Ed.). The nature of expertise. Hillsdale: Lawrence Erlbaum.

Hallam, S. (2001). The development of metacognition in musicians: Implications for education. British fournal of Music Education, 18(1), 27-39.

Lafortune, J. \& Hébert (2000). Pour guider la métacognition. Quebec: Presses de l'Université du Québec.

McPherson, G. E., \& Davidson, J. W. (2006). Playing an instrument. En G. E. McPherson (Ed.). The child as musician: A handbook of musical development. Nueva York: Oxford University Press.

Richer y otros. (2004). Outil d'évaluation de la métacognition : processus de validation et utilisation à des fins pédagogiques. En R. Pallascio, M. F. Daniel \& L. Lafortune (dir.). Pensée et réflexivité: théories et pratiques. Quebec: Presses de l'Université du Québec.

Saint-Pierre, L. (1994). La métacognition, qu'en est-il? Revue des sciences de l'éducation, 20(3), 529- 545.

Woody, R. H. (2001). Learning from the experts: applying research in expert performance to music education. Applications of Research in Music Education, 19(2), 9-14.

\section{Anexo A. Cuestionario COMÉGAM adaptado a la práctica instrumental}

\begin{tabular}{|l|c|c|c|c|c|}
\hline Cód. & $N^{\text {O }}$ & Ítem & TD D & DA & TDA \\
\hline CS & 1 & $\begin{array}{l}\text { Conozco las estrategias que } \\
\text { me ayudan a practicar } \\
\text { bien. }\end{array}$ & & & \\
\hline CP & 2 & $\begin{array}{l}\text { Puedo nombrar las } \\
\text { cualidades que tengo que } \\
\text { me ayudan a practicar } \\
\text { mejor. }\end{array}$ & & \\
\hline
\end{tabular}

\begin{tabular}{|c|c|c|c|c|c|c|}
\hline Cód. & $\mathrm{N}^{\mathrm{o}}$ & Ítem & $\mathrm{TD}$ & D & DA & TDA \\
\hline GAP & 3 & $\begin{array}{l}\text { Al principio de mis } \\
\text { prácticas, organizo las } \\
\text { informaciones que conozco } \\
\text { sobre los aspectos que debo } \\
\text { estudiar, las cuales me } \\
\text { pueden ayudar a practicar } \\
\text { mejor. }\end{array}$ & & & & \\
\hline GAC & 4 & $\begin{array}{l}\text { Cuando estoy practicando, } \\
\text { soy capaz de identificar mis } \\
\text { errores }\end{array}$ & & & & \\
\hline $\mathrm{CP}$ & 5 & $\begin{array}{l}\text { Conozco mis debilidades } \\
\text { principales en el } \\
\text { instrumento. }\end{array}$ & & & & \\
\hline GAC & 6 & $\begin{array}{l}\text { Durante mis prácticas, } \\
\text { vigilo la calidad de mi } \\
\text { práctica. }\end{array}$ & & & & \\
\hline C.S & 7 & $\begin{array}{l}\text { Puedo nombrar estrategias } \\
\text { eficientes para prepararme } \\
\text { a hacer una presentación } \\
\text { en público }\end{array}$ & & & & \\
\hline CT & 8 & $\begin{array}{l}\text { Durante mis prácticas, sé } \\
\text { reconocer el tipo de tarea } \\
\text { que ejecuto con facilidad. }\end{array}$ & & & & \\
\hline GAR & 9 & $\begin{array}{l}\text { Si no llego a resolver una } \\
\text { dificultad utilizando ciertas } \\
\text { estrategias, utilizo otras. }\end{array}$ & & & & \\
\hline GAP & 10 & $\begin{array}{l}\text { Antes de empezar a } \\
\text { estudiar, establezco los } \\
\text { aspectos que quiero } \\
\text { mejorar y las estrategias } \\
\text { con las que voy a hacerlo. }\end{array}$ & & & & \\
\hline GAC & 11 & $\begin{array}{l}\text { Durante mis prácticas, me } \\
\text { aseguro de comprender } \\
\text { bien lo que tengo que } \\
\text { hacer. }\end{array}$ & & & & \\
\hline C.S & 12 & $\begin{array}{l}\text { Conozco cuáles son las } \\
\text { estrategias más eficientes } \\
\text { para practicar. }\end{array}$ & & & & \\
\hline GAP & 13 & $\begin{array}{l}\text { Antes de empezar a } \\
\text { estudiar, evalúo la } \\
\text { magnitud de los aspectos } \\
\text { que debo mejorar. }\end{array}$ & & & & \\
\hline $\mathrm{CP}$ & 14 & $\begin{array}{l}\text { Puedo reconocer mis } \\
\text { puntos débiles cuando me } \\
\text { comparo con otros músicos } \\
\text { que tocan el mismo } \\
\text { instrumento que yo. }\end{array}$ & & & & \\
\hline GAR & 15 & $\begin{array}{l}\text { Durante mis prácticas, si } \\
\text { me doy cuenta de que } \\
\text { puedo utilizar una } \\
\text { estrategia más eficiente, } \\
\text { modifico mis acciones. }\end{array}$ & & & & \\
\hline
\end{tabular}




\begin{tabular}{|c|c|c|c|c|c|c|}
\hline Cód. & $\mathrm{N}^{\mathrm{o}}$ & Ítem & $\mathrm{TD}$ & D & $\mathrm{DA}$ & TDA \\
\hline CT & 16 & $\begin{array}{l}\text { Reconozco las tareas que } \\
\text { necesitan una preparación } \\
\text { más elaborada. }\end{array}$ & & & & \\
\hline GAR & 17 & $\begin{array}{l}\text { Si no puedo arreglar una } \\
\text { dificultad, vuelvo a } \\
\text { comenzar buscando otras } \\
\text { maneras de proceder }\end{array}$ & & & & \\
\hline GAC & 18 & $\begin{array}{l}\text { Me cuestiono sobre mi } \\
\text { manera de estudiar } \\
\text { durante mis prácticas. }\end{array}$ & & & & \\
\hline GAP & 19 & $\begin{array}{l}\text { Si tengo que trabajar un } \\
\text { aspecto específico (técnica, } \\
\text { musicalidad, } \\
\text { memorización, etc.) planeo } \\
\text { cómo hacerlo reuniendo la } \\
\text { información disponible } \\
\text { sobre ello. }\end{array}$ & & & & \\
\hline GAC & 20 & $\begin{array}{l}\text { Cuando estudio un aspecto } \\
\text { específico, intento } \\
\text { reconocer mis errores. }\end{array}$ & & & & \\
\hline CS & 21 & $\begin{array}{l}\text { Conozco ciertas estrategias } \\
\text { que pueden ayudarme a } \\
\text { practicar mejor. }\end{array}$ & & & & \\
\hline GAR & 22 & $\begin{array}{l}\text { Corrijo mi forma de } \\
\text { proceder si me doy cuenta } \\
\text { de que ésta es ineficiente. }\end{array}$ & & & & \\
\hline GT & 23 & $\begin{array}{l}\text { Durante mis prácticas, } \\
\text { puedo identificar el nivel } \\
\text { de dificultad de los aspectos } \\
\text { que debo estudiar. }\end{array}$ & & & & \\
\hline GAR & 24 & $\begin{array}{l}\text { Si una manera de proceder } \\
\text { no da los resultados } \\
\text { previstos, trato de } \\
\text { encontrar otros medios } \\
\text { para alcanzar mis } \\
\text { objetivos. }\end{array}$ & & & & \\
\hline CP & 25 & $\begin{array}{l}\text { Soy capaz de nombrar mis } \\
\text { cualidades cuando me } \\
\text { comparo con otros } \\
\text { estudiantes. }\end{array}$ & & & & \\
\hline GAC & 26 & $\begin{array}{l}\text { Durante mis periodos de } \\
\text { estudio, tomo pausas para } \\
\text { preguntarme si voy por el } \\
\text { buen camino. }\end{array}$ & & & & \\
\hline CT & 27 & $\begin{array}{l}\text { Puedo identificar cuáles } \\
\text { son los aspectos que puedo } \\
\text { estudiar fácilmente. }\end{array}$ & & & & \\
\hline $\mathrm{CP}$ & 28 & $\begin{array}{l}\text { Reconozco en qué soy } \\
\text { bueno cuando toco. }\end{array}$ & & & & \\
\hline CT & 29 & $\begin{array}{l}\text { Puedo nombrar qué tareas } \\
\text { necesitan soluciones más } \\
\text { exigentes durante mis } \\
\text { periodos de estudio. }\end{array}$ & & & & \\
\hline
\end{tabular}

\begin{tabular}{|c|c|c|c|c|c|c|}
\hline Cód. & $\mathrm{N}^{\mathrm{o}}$ & Ítem & TD & D & $\mathrm{DA}$ & TDA \\
\hline GAR & 30 & $\begin{array}{l}\text { Si no logro mi objetivo, } \\
\text { evalúo lo que dio, o no, } \\
\text { buenos resultados en mi } \\
\text { forma de practicar. }\end{array}$ & & & & \\
\hline C.S & 31 & $\begin{array}{l}\text { Puedo enumerar varias } \\
\text { maneras de memorizar mi } \\
\text { repertorio. }\end{array}$ & & & & \\
\hline CS & 32 & $\begin{array}{l}\text { Puedo enumerar diferentes } \\
\text { formas de estudiar un } \\
\text { mismo aspecto. }\end{array}$ & & & & \\
\hline GAP & 33 & $\begin{array}{l}\text { Antes de empezar a } \\
\text { estudiar, examino los } \\
\text { aspectos que debo } \\
\text { practicar. }\end{array}$ & & & & \\
\hline $\mathrm{CP}$ & 34 & $\begin{array}{l}\text { Puedo nombrar mis } \\
\text { cualidades que hacen que } \\
\text { sea eficiente cuando } \\
\text { estudio. }\end{array}$ & & & & \\
\hline CT & 35 & $\begin{array}{l}\text { Puedo describir los } \\
\text { aspectos de mi práctica } \\
\text { instrumental que requieren } \\
\text { más concentración durante } \\
\text { mi estudio. }\end{array}$ & & & & \\
\hline GAP & 36 & $\begin{array}{l}\text { Antes de empezar a } \\
\text { estudiar, determino lo que } \\
\text { voy a hacer. }\end{array}$ & & & & \\
\hline
\end{tabular}

Leyenda: Cód.: código; TD: totalmente en desacuerdo; D: en desacuerdo; DA: de acuerdo; TDA: totalmente de acuerdo.

Significado códigos (no deben darse al alumno mientras responde el cuestionario).

- Conocimientos metacognitivos.

- CP: conocimientos acerca de las personas.

- CT: de las tareas.

- CS: de las estrategias.

- Gestión de la actividad mental.

- GAP: Planificación.

- GAC: Control.

- GAR: Regulación.

\section{Anexo B. Fichas de reflexión.}

\begin{tabular}{|l|l|}
\hline \multicolumn{2}{|c|}{ Ficha 1} \\
\multicolumn{2}{|c|}{ Reflexiones acerca de las estrategias } \\
Preguntas $1-7-12-31-32$
\end{tabular}




\begin{tabular}{|c|c|}
\hline \multicolumn{2}{|c|}{$\begin{array}{l}\text { Ficha } 2 \\
\text { Reflexiones acerca de las personas } \\
\text { Preguntas 2-5-14-25-28-34 }\end{array}$} \\
\hline $\begin{array}{l}\text { Si la mayoría de las veces } \\
\text { contestaste totalmente de acuerdo } \\
\text { o de acuerdo: } \\
\text { - ¿Cuáles son tus fortalezas } \\
\text { cualidades respecto a la } \\
\text { ejecución instrumental? } \\
\text { - ¿Cuáles son tus debilidades } \\
\text { respecto a la ejecución } \\
\text { instrumental? } \\
\text { - Da un ejemplo específico } \\
\text { de una pieza en la que has } \\
\text { identificado una de tus } \\
\text { fortalezas. } \\
\text { - Da un ejemplo específico } \\
\text { de una pieza en la que has } \\
\text { identificado uno de tus } \\
\text { debilidades. }\end{array}$ & $\begin{array}{l}\text { Si la mayoría de las veces } \\
\text { contestaste en desacuerdo o } \\
\text { totalmente en desacuerdo: } \\
\text { Piensa en una práctica que } \\
\text { haya sido muy eficiente y otra } \\
\text { que no. Describe brevemente } \\
\text { estas dos situaciones. } \\
\text { - ¿Qué ha hecho que tu } \\
\text { práctica haya sido eficiente? } \\
\text { - ¿Has actuado de una } \\
\text { manera especial? } \\
\text { - ¿Qué pensamientos tuviste } \\
\text { en esos momentos? } \\
\text { - ¿Cuál es tu parte de } \\
\text { responsabilidad en el éxito } \\
\text { de esta primera situación? } \\
\text { Vuelve a realizar el mismo } \\
\text { ejercicio con tu práctica menos } \\
\text { eficiente. }\end{array}$ \\
\hline \multicolumn{2}{|c|}{$\begin{array}{c}\text { Ficha } 3 \\
\text { Reflexiones acerca de las tareas } \\
\text { Preguntas 8-16-23-27-29-35 }\end{array}$} \\
\hline $\begin{array}{l}\text { Si la mayoría de las veces } \\
\text { contestaste totalmente de acuerdo } \\
\text { o de acuerdo: } \\
\text { - ¿Cuáles son los tipos de } \\
\text { tareas que debes realizar } \\
\text { durante tus periodos de } \\
\text { estudio que son más fáciles } \\
\text { para ti? ¿Por qué? } \\
\text { - ¿Cuáles son las tareas que } \\
\text { requieren más } \\
\text { concentración? } \\
\text { • ¿Cuáles son las tareas que } \\
\text { requieren un mayor } \\
\text { esfuerzo? ¿Por qué? }\end{array}$ & $\begin{array}{l}\text { Si la mayoría de las veces } \\
\text { contestaste en desacuerdo o } \\
\text { totalmente en desacuerdo: } \\
\text { - Menciona una serie de } \\
\text { tareas diferentes (al menos 5) } \\
\text { que debes realizar mientras } \\
\text { estudias. Describe } \\
\text { brevemente cada una de } \\
\text { estas tareas. Identifica la que } \\
\text { te parezca más fácil. } \\
\text { - Trata de averiguar por qué } \\
\text { esta tarea es fácil (¿requiere } \\
\text { menos concentración?, } \\
\text { ¿menor destreza?...). } \\
\text { - Marca la tarea que te } \\
\text { parezca más difícil. } \\
\text { - Identifica la tarea que te } \\
\text { parezca más difícil. } \\
\text { - Examina las otras tres tareas } \\
\text { y trata de encontrar si tienen } \\
\text { puntos en común. Trata } \\
\text { también de identificar sus } \\
\text { diferencias. }\end{array}$ \\
\hline
\end{tabular}

Ficha 4

Reflexiones sobre sus conocimientos metacognitivos Vuelve a leer tus respuestas a las 3 primeras fichas.

Etapa 1:

- Con un marcador, subraya los puntos que creas que son importantes conocer acerca de ti mismo en una situación de aprendizaje (cuando estudias tu instrumento).

- Si algunas de las cosas que escribiste te sorprendieron, enciérralas en un círculo.

- Si haces reflexiones sobre algunas de las cosas que escribiste, anótalas en el margen.

Etapa 2:

- Haz un una síntesis de lo que has cerrado en círculos.

- Haz una síntesis de lo que has subrayado con el marcador.

- Escribe las reflexiones que surgieron durante la lectura de tus fichas.



Si la mayoría de las veces contestaste totalmente de acuerdo

o de acuerdo:

- Enumera las principales acciones que realizas para planear tu estudio instrumental.

- Indica los primeros pensamientos que te vienen a la mente cuando empiezas a estudiar.
Si la mayoría de las veces contestaste en desacuerdo o totalmente en desacuerdo:

- Pensando en tu práctica instrumental, describe cómo podrías elaborar un plan antes de empezar a estudiar.

- ¿Crees que te puede ser útil respetar este plan? ¿Por qué?

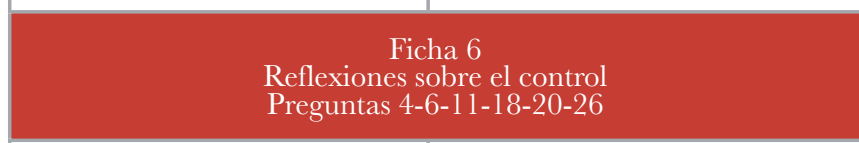

Si la mayoría de las veces contestaste totalmente de acuerdo o de acuerdo:

- Menciona ejemplos concretos de lo que haces para evaluar el logro de tus objetivos cuando estudias.

- ¿Qué haces para evaluar tus avances mientras estudias?

- Después de practicar, ¿qué haces para evaluar la eficacia de tu estudio?

- ¿Cómo vas a asegurarte de que estás haciendo lo correcto mientras estudias? Da ejemplos concretos.
Si la mayoría de las veces contestaste en desacuerdo o totalmente en desacuerdo:

- Describe las cosas que podrías hacer para asegurarte de que estás haciendo lo correcto. Si te cuesta trabajo, piensa en lo que haría un/a amigo/a en tu lugar.

- ¿Por qué no sueles evaluar tu trabajo durante y después de estudiar?

- ¿Crees que te sería útil detenerte a evaluar tus acciones o formas de proceder? Si respondiste sí, ¿por qué no lo haces de manera espontánea? Si respondiste no, ¿por qué esta manera de funcionar no te conviene? ¿Qué podría ayudarte?

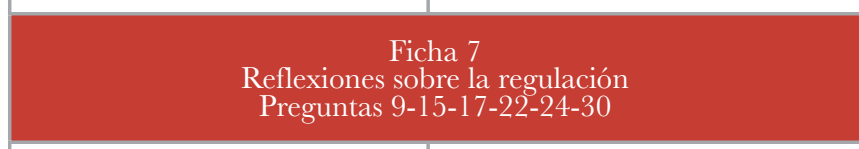

Si la mayoría de las veces contestaste totalmente de acuerdo o de acuerdo:

- Menciona una dificultad que te hizo estudiar de diferentes maneras

- ¿Qué te hizo cambiar de forma de proceder?

- ¿Modificar tus acciones fue positivo para ti? Si respondiste sí, ¿qué beneficio obtuviste? Si respondiste no, ¿cambiaste otras maneras de proceder después? ¿Cuáles fueron esos cambios?
Si la mayoría de las veces contestaste en desacuerdo o totalmente en desacuerdo:

- Describe brevemente una práctica en la que no te hayas sentido cómodo.

- ¿Qué te hubiera permitido estar más cómodo durante esta práctica?

- ¿Qué podrías hacer para tener más posibilidades de lograr tus objetivos?

- ¿Qué correctivos podrías aplicar a tu estudio instrumental? 
Etapa 1:

- Con un marcador, subraya las acciones que te traen beneficios o ventajas en términos de estudio instrumental.

- Si algunas de las cosas que escribiste no son "naturales", es decir, que no haces de forma espontánea, enciérralas en un círculo.

- Si algunas de las cosas que escribiste son "naturales" para ti, es decir, que haces espontáneamente, dibuja una señal en el margen.

Etapa 2:

- ¿Cuáles son las ventajas o beneficios de las acciones que subrayaste con el marcador?

- Haz una síntesis de lo que encerraste, trata de reformular y de explicar los beneficios que obtuviste.

- Escribe las reflexiones que surgieron durante la lectura de tus fichas. 


\section{Sobre los Autores}

\section{Malinalli Peral García}

Guitarrista de formación clásica, obtuvo una Licenciatura en Interpretación Musical por la Universidad de Sherbrooke (2005) y una Maestría en Didáctica Instrumental por la de Laval (2008), las dos con Mención de Excelencia. Sus estudios de Maestría fueron subvencionados por el FONGA (Fondo Nacional para la Cultura y las Artes) y por el Gobierno del Estado de Veracruz, México. Becaria del CRSH (Canadá), realiza actualmente un Doctorado en Educación Musical en la Universidad Laval. Sus intereses de investigación se centran en el desarrollo de la metacognición durante el aprendizaje de un instrumento musical.

\section{Francis Dubé}

Miembro titular del OICRM, es Profesor Asociado de Didáctica Instrumental en la Facultad de Música de la Universidad Laval. Pianista de formación, obtuvo una licenciatura y maestría en interpretación en la Universidad de Montreal, posteriormente continuó realizando estudios superiores en París. Financiado por el FRQ-SC y el CRSH para realizar sus trabajos de investigación, es corresponsable del Laboratorio de Investigación en formación auditiva y didáctica instrumental (www.larfadi.oicrm.org); así mismo, supervisa a más de 25 estudiantes de segundo y tercer ciclo (http:// centreexcellence.oicrm.org/membres/francis-dube).

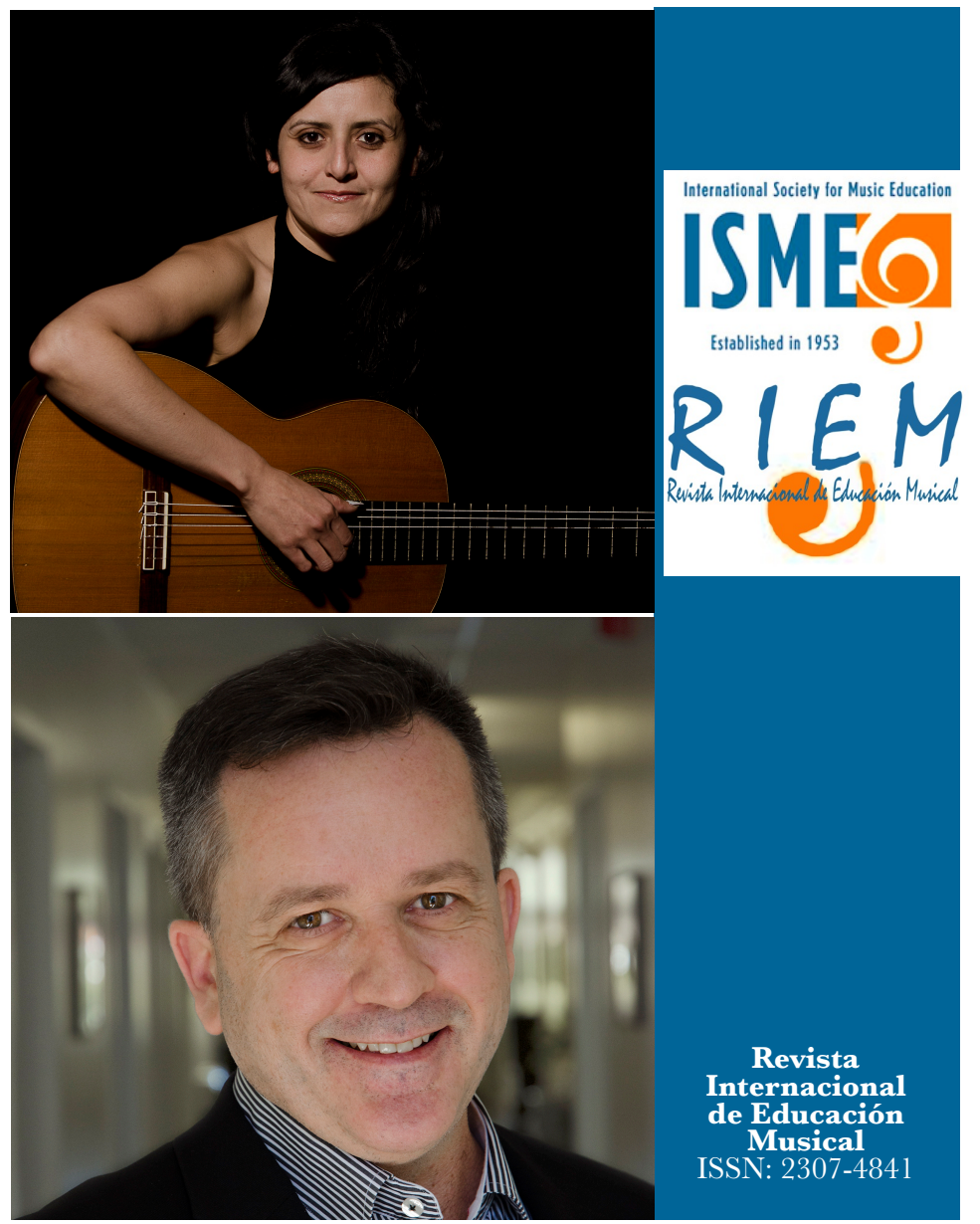

\section{EQUIPO EDITORIAL}

Editor:

José Luis Aróstegui Plaza, Universidad de Granada (España)

\section{Editora Adjunta:}

Rosa María Serrano Pastor, Universidad de Zaragoza (España)

\section{Consejo Editorial}

María del Carmen Aguilar, Instituto Coral de Buenos Aires (Argentina)

Miquel Alsina Tarrés, Universidad de Gerona (España)

Graça Boal Palheiros, Instituto de Educación de Oporto (Portugal) Rubén Gaztambide Fernández, Universidad de Toronto (Canadá)

Patricia Adelaida González, Universidad Autónoma de Chihuahua (México)

Claudia Gluschankof, Instituto Levinsky (Israel)

María Cecilia Jorquera Jaramillo, Universidad de Sevilla (España)

Gotzon Ibarretxe Txakartegi, Universidad del País Vasco (España)

Yore Kedem, Universidad de Illinois (Estados Unidos)

Saville Kushner, Universidad de Auckland (Nueva Zelanda)

Ana Laucirica Larrinaga, Universidad Pública de Navarra (España)

Ana Lucia Louro, Universidad Federal de Santa María (Brasil)

Isabel Cecilia Martínez, Universidad Nacional de La Plata (Argentina)
Teresa Mateiro, Universidad del Estado de Santa Catarina (Brasil)

María Teresa Moreno, Universidad Laval (Canadá)

Graça Mota, Instituto de Educación de Oporto (Portugal)

Oscar Odena, Universidad de Glasgow (Reino Unido)

Gabriel Enrique Rusinek Milner, Universidad Complutense de Madrid (España)

Patricia Sabbatella Riccardi, Universidad de Cádiz (España)

Favio Shifres, Universidad Nacional de La Plata (Argentina)

Christopher Suazo, Colegio Americano de Madrid (España)

Maria dels Àngels Subirats Bayego, Universidad de Barcelona (España)

António Ângelo Ferreira Vasconcelos, Instituto de Educación de Setúbal (Portugal)

Gloria Patricia Zapata Restrepo, Universidad de Antioquía (Colombia) 